\title{
Prevalência de retinopatia da prematuridade em prematuros atendidos no período de 1992-1999 em Joinville (SC): avaliação de riscos associados - "screening"
}

\author{
Prevalenceof retinopathy of prematurity in premature babies examined during the \\ period 1992-1999, Join ville(SC):evaluation of associated risks-screening
}

\author{
Ligia Beatriz Bonotto' \\ Ana Tereza Ramos Moreira ${ }^{2}$ \\ Denise Siqueira Carvalho ${ }^{3}$
}

Descritores: Prematuro; Retinopatia da prematuridade/epidemiologia; Fatores de risco

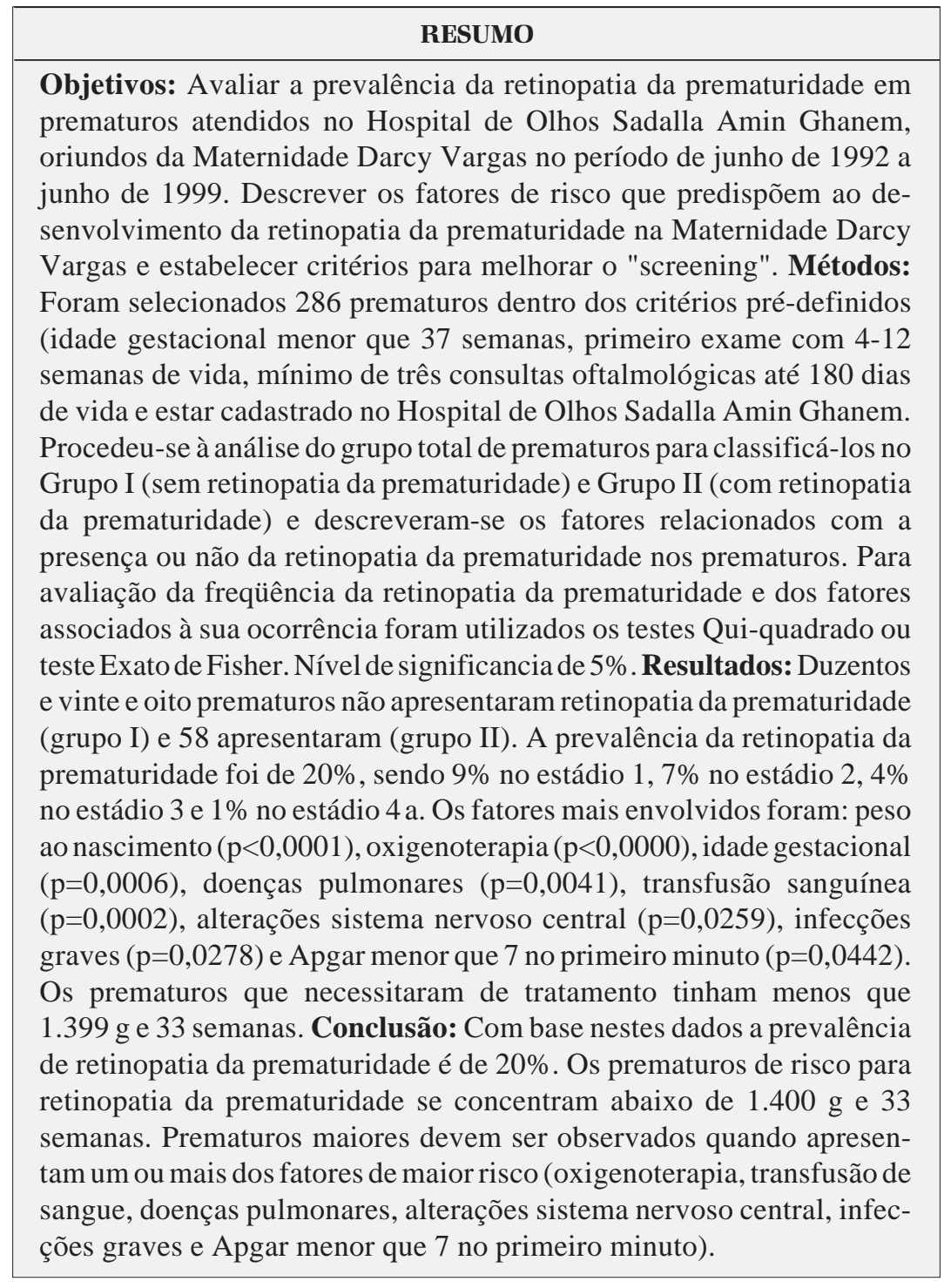

Objetivos: Avaliar a prevalência da retinopatia da prematuridade em prematuros atendidos no Hospital de Olhos Sadalla Amin Ghanem, oriundos da Maternidade Darcy Vargas no período de junho de 1992 a junho de 1999. Descrever os fatores de risco que predispõem ao desenvolvimento da retinopatia da prematuridade na Maternidade Darcy Vargas e estabelecer critérios para melhorar o "screening". Métodos: Foram selecionados 286 prematuros dentro dos critérios pré-definidos (idade gestacional menor que 37 semanas, primeiro exame com 4-12 semanas de vida, mínimo de três consultas oftalmológicas até 180 dias de vida e estar cadastrado no Hospital de Olhos Sadalla Amin Ghanem. Grupo I (sem retinopatia da prematuridade) e Grupo II (com retinopatia da prematuridade) e descreveram-se os fatores relacionados com a avaliação da freqüência da retinopatia da prematuridade e dos fatores associados à sua ocorrência foram utilizados os testes Qui-quadrado ou e vinte e oito prematuros não apresentaram retinopatia da prematuridade (grupo I) e 58 apresentaram (grupo II). A prevalência da retinopatia da prematuridade foi de $20 \%$, sendo $9 \%$ no estádio $1,7 \%$ no estádio $2,4 \%$ no estádio 3 e $1 \%$ no estádio 4 a. Os fatores mais envolvidos foram: peso ao nascimento $(\mathrm{p}<0,0001)$, oxigenoterapia $(\mathrm{p}<0,0000)$, idade gestacional $(\mathrm{p}=0,0006)$, doenças pulmonares $(\mathrm{p}=0,0041)$, transfusão sanguínea $(\mathrm{p}=0,0002)$, alterações sistema nervoso central $(\mathrm{p}=0,0259)$, infecções graves $(\mathrm{p}=0,0278)$ e Apgar menor que 7 no primeiro minuto $(\mathrm{p}=0,0442)$. Os prematuros que necessitaram de tratamento tinham menos que 1.399 g e 33 semanas. Conclusão: Com base nestes dados a prevalência retinopatia da prematuridade se concentram abaixo de 1.400 g e 33 semanas. Prematuros maiores devem ser observados quando apresenam um ou mais dos fatores de maior risco (oxigenoterapia, transfusão de ções graves e Apgar menor que 7 no primeiro minuto).
\end{abstract}

\footnotetext{
Trabalho realizado na Maternidade Darcy Vargas Joinville (SC).

${ }^{1}$ Mestre, Setor de Ciências da Saúde, Universidade Federal do Paraná - UFPR - Curitiba (PR) - Brasil. Médica Oftalmologista no Hospital de Olhos Sadalla Amin Ghanem - Joinville (SC) - Brasil.

${ }^{2}$ Professora Adjunta do Departamento de Oftalmologia da UFPR - Curitiba (PR) - Brasil.

${ }^{3}$ Professora Adjunta do Departamento de Saúde Comunitária da UFPR - Curitiba (PR) - Brasil.

Endereço para correspondência: Rua Abdom Batista, 172 - Joinville (SC) CEP 89201-010

E-mail: ligia@oftalmopediatria.com.br

Recebido para publicação em 23.06.2004

Última versão recebida em 09.02.2006

Aprovação em 26.06.2006
} 
56 Prevalência de retinopatia da prematuridade em prematuros atendidos no período de 1992-1999 em Joinville (SC): avaliação de riscos associados - "screening"

\section{INTRODUÇÃOO}

A retinopatia da prematuridade (ROP) é uma doença vasoproliferativa que ocorre em recém-nascidos prematuros. Nestes bebês, por ocasião do nascimento, a vasculogênese da retina está incompleta favorecendo a formação de tecido neovascular. Na grande maioria dos casos, este tecido sofre involução espontânea, enquanto, no restante, evolui para uma proliferação fibrovascular em direção ao vítreo, formando membranas e trações retinianas. O descolamento da retina pode ser decorrente dessas trações, acarretando nesses olhos um baixo poder de resolução visual ${ }^{(1)}$. A ROP foi primeiramente estudada e descrita por Terry em $1942^{(2)}$. Seguiram-se outros autores, nesta mesma década, que observaram melhor o desenvolvimento da doença e conseguiram percebê-la após o nascimento, assim como puderam diferenciá-la de outras doenças do vítreo ${ }^{(3-4)}$.

Em 1948, nos Estados Unidos, a ROP foi responsável por $30 \%$ das causas de amaurose observadas nas crianças préescolares $^{(5)}$. No Brasil não é conhecida a importância da ROP como causa de amaurose até os dias de hoje. E o estudo desta doença ainda é iniciativa isolada em alguns serviços públicos ou particulares ${ }^{(6)}$.

A aceitação da classificação internacional da ROP (19841987) ajudou imensamente a reconhecer cientificamente, em linguagem única, as mudanças vasculares que ocorrem nas retinas dos prematuros, assim como estabelecer parâmetros para o seu tratamento ${ }^{(7-9)}$.

Vários fatores etiológicos foram associados ao desenvolvimento da ROP como peso ao nascimento e idade gestacional baixos, infecções, deficiência de vitamina E e A, exposição a luz entre outras ${ }^{(5,7,10)}$.

Até 1992, Joinville não contava com nenhum acompanhamento oftalmológico de rotina e nenhuma maternidade. Considerando-se a importância da ROP e conhecendo-se os riscos que os prematuros estavam correndo, foi desenvolvido o presente estudo na Maternidade Darcy Vargas (MDV) com os seguintes objetivos:

1) Avaliar a prevalência da ROP em prematuros atendidos no Hospital de Olhos Sadalla Amin Ghanem, oriundos da Maternidade Darcy Vargas - Joinville (SC).

2) Descrever os fatores de risco que predispõem ao desenvolvimento da ROP na MDV.

3) Identificar o grupo de maior risco para melhorar o "screening".

4) Selecionar os prematuros em Grupo I sem ROP e Grupo II com ROP.

\section{MÉTODOS}

É um estudo observacional, prospectivo e analítico dos prematuros nascidos e internados na Maternidade Darcy Vargas em Joinville (SC), que foram acompanhados para avaliação da ROP no período de junho de 1992 a junho de 1999.
Dentre os prematuros nascidos na MDV, no referido período foram selecionados os que atendiam os critérios de inclusão como peso ao nascimento menor ou igual a $1.600 \mathrm{~g}$ ou idade gestacional menor que 37 semanas, o prematuro deveria ter o mínimo de seis meses de idade até dezembro de 1999, o primeiro exame oftalmológico com quatro semanas de vida, mínimo de três consultas oftalmológicas até 180 dias de vida e estar cadastrado no Hospital de Olhos Sadalla Amin Ghanem (HOSAG). Os critérios de exclusão foram os óbitos, acima de 12 semanas para primeiro exame, ausência de informação sobre a mãe, ausência de dados do período neonatal e os casos que não apresentaram dados da consulta oftalmológica (descrição do mapeamento de retina).

A seleção dos prematuros para exame de risco de desenvolver ROP sempre foi feita pela equipe da oftalmologia, composta de oftalmologista pediátrica e sua auxiliar. Os prematuros dentro dos critérios já referidos foram listados usando as iniciais RN (recém-nascido) seguidas do nome da mãe e do prematuro. Nesta lista constavam ainda a data da triagem, a data prevista para o primeiro e segundo exames. A lista serviu de controle na hora de protocolar os prematuros no programa de computador no HOSAG. Programa criado exclusivamente para os dados dos prematuros e de suas mães. Neste banco as informações acompanham o prematuro desde seu primeiro contato com a oftalmologista na maternidade até a necessidade de óculos, tratamento para correção de estrabismo, acompanhamento no setor de visão, exames de avaliação subseqüentes têm campos criados para seu devido preenchimento.

Os olhos foram dilatados com uma gota de tropicamida a $0,5 \%$, por duas vezes com intervalo de dez minutos, o exame oftalmológico era feito dez minutos após. Raras vezes foi necessária dose adicional. Quando havia dificuldade na dilatação, foi considerado sinal plus ${ }^{(11)}$ e repetia-se o exame na semana seguinte. Com essas medidas de segurança usou-se um só tipo de colírio midriático diferente do que se preconiza na literatura(12-14).

O exame de mapeamento de retina era realizado com oftalmoscópio indireto, lente de 20 dioptrias, blefarostato e depressor escleral, quando necessário. O exame oftalmológico sempre foi realizado pela mesma oftalmologista.

O primeiro exame era agendado para quatro semanas após data do nascimento, o segundo, 15 dias após e os subseqüentes eram agendados de dois em dois meses até seis meses e o exame de grau agendado para 12 meses de idade. Durante este esquema foram observados outros transtornos como estrabismo. Na presença de retinopatia da prematuridade, reduzia-se o intervalo para menos de uma semana ${ }^{(15-16)}$.

As crianças muito prematuras foram examinadas, geralmente, mais de quatro vezes na própria maternidade onde estavam internadas.

$\mathrm{Na}$ alta hospitalar, a caderneta de saúde do prematuro continha orientações para agendamento dos exames subseqüentes no HOSAG. Alguns casos foram encaminhados para exame oftalmológico, diretamente pelo pediatra. Nos retornos ambulatoriais, o resultado do exame e a data da nova visita 
sempre foi anotada na caderneta de saúde, assim o pediatra podia acompanhar o desenvolvimento ocular da criança e ajudar na ênfase aos novos retornos.

A classificação da Retinopatia da Prematuridade obedeceu aos critérios estabelecidos pelos Comitês Internacionais de 1984 e 1987 e o tratamento obedeceu aos critérios estabelecidos pelo "Cryotherapy for Retinopaty of Prematurity Cooperative Group" e outras publicações da época. A crioterapia ou laser foi realizado em casos de ROP estádio 3 com plus, zona 2 com cinco horas contínuas ou a soma de oito horas ou mais, descontínuas. Realizou-se a retinopexia nos casos com estádio $4 \mathrm{a}^{(8-9,17-19)}$.

Para o presente estudo foram extraídos, do banco de dados geral, acima referido, informações dos prematuros que preencheram os critérios de inclusão. Os dados extraídos formaram, no Excel, um novo banco com informações para esta análise. As seguintes informações foram avaliadas: identificação, sexo, dados do nascimento como peso em gramas, idade gestacional em semanas (método de Capurro); doença ou alteração do trato respiratório desde apnéia, taquipnéia, membrana hialina, infecção respiratória, pneumonia. Condições de manipulação, como drenagem pleural e oxigenioterapia; alterações do sistema cardíaco quaisquer alterações de funcionamento de válvula ou tipo de comunicação intraventricular ou atrial; alterações ou doenças do sistema digestivo, enterocolites necrotisantes, atresia de jejuno, estase com distenção gástrica e outras; alterações do sistema nervoso central, foram consideradas hemorragia periventricular, hemorragia intraventricular e hemorragia intracraniana; doenças metabólicas, foram consideradas a hipo ou hipercalcemia, hipo ou hipernatremia, hipo ou hiperglicemia, alcalose ou acidose metabólicas. Infecções graves, foram consideradas septicemia, meningite, enterocolite, pneumonia por pseudomonas; toxoplasmose; outras doenças, foram consideradas icterícia, hiperbilirrubinemia, infecção perinatal, conjuntivite, alteração no tamanho do fígado, anemia; anomalias congênitas, foram consideradas as hérnias inguinais e umbilicais, micronactia, orelha em concha e apêndices cutâneos, estenose de artéria pulmonar, síndrome de West, síndrome de Down e outras. Os dados foram obtidos do quadro de malformações e do quadro de outras doenças do banco de dados geral.

Todas as variáveis acima foram estudadas como variáveis qualitativas simples, sim ou não.

Quanto ao tratamento necessário durante a internação na maternidade foram consideradas: Oxigenioterapia, fototerapia e transfusão de sangue, os dados foram obtidos dos quadros específicos para cada tratamento mencionado no banco de dados geral. As variáveis foram estudadas como variáveis qualitativas simples, sim ou não.

E quanto ao critério de diagnóstico da retinopatia da prematuridade, utilizou-se os códigos: zero- sem retinopatia; 1- retinopatia estádio 1;2-retinopatia estádio 2; 3-retinopatia estádio 3; 4- retinopatia estádio 4 a.

Para análise dos fatores de risco, os prematuros sem ROP foram classificados no grupo I e os prematuros com ROP no grupo II.
Os gêmeos foram analisados da mesma maneira que os não gêmeos, quando não tinham retinopatia eram incluídos no grupo I e quando tinham retinopatia em algum estádio, eram incluídos no grupo II.

Para a classificação da retinopatia da prematuridade foi considerado o olho de maior estádio.

Para a análise estatística foram utilizados testes estatísticos de significância, quando os pressupostos para a utilização do teste Qui-quadrado eram verificados utilizou-se este teste para a comparação entre os dois grupos; crianças sem retinopatia da prematuridade (grupo I) e com retinopatia (grupo II). Nos casos em que a utilização do teste Qui-quadrado não era adequado, utilizou-se o teste Exato de Fisher. O nível de significância foi de $5 \%$.

\section{RESULTADOS}

Nasceram, durante o período de junho de 1992 a junho de 1999, na Maternidade Darcy Vargas, 45.849 crianças. Destas, $1.422(3,1 \%)$ eram prematuras, e compreenderam a população de referência do estudo.

Foram a óbito 256 durante a internação na UTI neonatal 200 prematuros não sobreviveram mais de sete dias.

Dos 1.166 prematuros sobreviventes, 457 não retornaram para seguimento após a alta da maternidade, sendo que 709 foram protocolados no serviço ambulatorial de seguimento ao prematuro no HOSAG.

Excluindo, os não cadastrados, os óbitos e os 423 prematuros que não preencheram os critérios de seleção descritos na metodologia, permanecem para análise estatística 286 prematuros e 269 mães. Dentre o grupo de prematuro selecionado, 58 desenvolveram algum grau de ROP, prevalência de $20 \%$. ROP com indicação de tratamento na população estudada seria de $2 \%$. A prevalência de cada estágio também está representada no gráfico 1.

Sexo: Dos 286 prematuros $162(56,64 \%)$ eram do sexo feminino: $36(22,2 \%)$ com ROP. Cento e vinte e quatro $(43,35 \%)$ eram do sexo masculino: $22(17,7 \%)$ com ROP.

$\mathrm{O}$ fator sexo não teve significado estatístico ( $\mathrm{p}=0,3504)$.

Peso ao nascimento: $O$ peso mínimo foi de $600 \mathrm{~g}$ e máximo $2.970 \mathrm{~g}$, peso médio $1.670 \mathrm{~g} \pm 474,9$. A relação peso ao nascimento com o grupo I e grupo II está na tabela 1.

$\mathrm{O}$ fator peso ao nascimento mostrou-se altamente significativo para o desenvolvimento da ROP. Apenas um caso dentre os prematuros sem ROP (grupo I) não teve a informação do peso ao nascimento.

Idade gestacional: A idade mínima encontrada foi de 23 semanas e máxima 36 semanas (média $31,9 \pm 4,155$ ). Três casos do grupo sem ROP (grupo I) não tinham essa informação. A idade gestacional mostrou-se altamente significativa em relação à ROP, p=0,0006. (Tabela 2).

O quadro 1 mostra todos os outros fatores, estudados nos prematuros com e sem ROP, com diferença estatisticamente significante. $\mathrm{O}$ quadro 2 mostra todos os fatores estudados 
58 Prevalência de retinopatia da prematuridade em prematuros atendidos no período de 1992-1999 em Joinville (SC): avaliação de riscos associados - "screening"

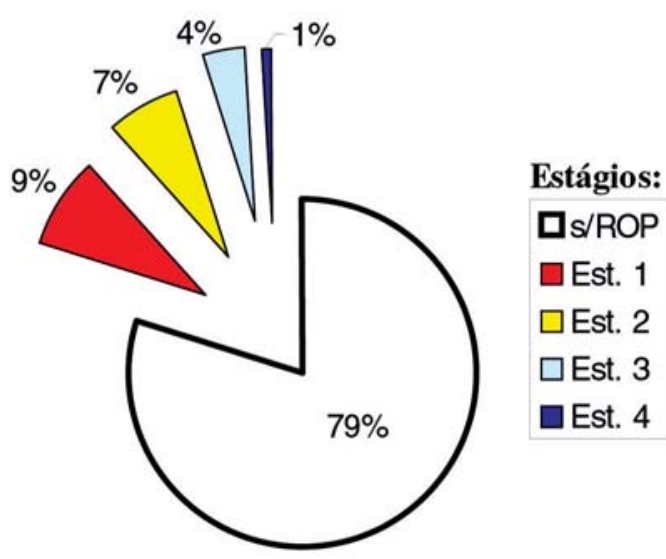

Fonte: Pesquisa de campo

Gráfico 1 - Distribuicão da ROP nos diferentes estágios entre os 286 prematuros. Maternidade Darcy Vargas - Joinville (SC). 1992 - 1999.

Tabela 1. Peso ao nascimento, em gramas dos prematuros com e sem ROP. Maternidade Darcy Vargas - Joinville (SC). 1992 - 1999

$\begin{array}{ccr}\text { Peso ao nascimento }(\mathbf{g}) & \text { Sem ROP } & \text { Com ROP } \\ 600-1.199 & 25(11,0 \%) & 21(36,2 \%) \\ 1.200-1.799 & 98(43,2 \%) & 27(46,6 \%) \\ 1.800-2.399 & 88(38,8 \%) & 9(15,5 \%) \\ 2.400> & 16(7,0 \%) & 1(1,7 \%) \\ \text { Total } & 227(100,0 \%) & 58(100,0 \%) \\ \text { Fonte: Pesquisa de campo; }(\mathrm{p}=9,58 \mathrm{E}-07 \text { ou } \mathrm{p}<0,0001) & \end{array}$

Tabela 2. Prematuros no Grupo I e II versus idade gestacional em semanas. Maternidade Darcy Vargas - Joinville (SC). 1992 - 1999

\begin{tabular}{crr} 
Idade gestacional (sem.) & Sem ROP & Com ROP \\
$23-27$ & $7(3,1 \%)$ & $5(8,6 \%)$ \\
$28-32$ & $95(42,2 \%)$ & $37(63,8 \%)$ \\
$33-36$ & $123(54,7 \%)$ & $16(27,6 \%)$ \\
Total & $225(100,0 \%)$ & $58(100,0 \%)$ \\
Fonte: Pesquisa de campo & & \\
\hline
\end{tabular}

nos prematuros com e sem ROP, sem diferença estatística importante, exceto sexo já analisado no início deste capítulo.

Dos 58 que desenvolveram a doença, $43 \%$ desenvolveram estágio $1 ; 34,5 \%$ estágio $2 ; 19 \%$ estágio 3 e 3,5\% estágio $4 a$.

No grupo estudado não foi encontrado estágio $4 \mathrm{~b}$ e 5 . (Gráficos 2 e 3).

Dos 58 prematuros com ROP, $90 \%$ regrediram espontaneamente e 10\% (6 casos) necessitaram de tratamento. Destes, quatro necessitaram crioterapia ou laserterapia, o prematuro de código 150, com PN de $1.090 \mathrm{~g}$ e IG de 28 semanas. O prematuro 161 , com PN de $1.350 \mathrm{~g}$ e IG de 35 semanas. A prematura $431 \mathrm{com}$ PN de $1.280 \mathrm{~g}$ e IG de 31 semanas. A prematura 505 com PN de $1.020 \mathrm{~g}$ e IG de 32 semanas.

Dois casos necessitaram de retinopexia mais crioterapia ou

\begin{tabular}{|c|c|c|c|}
\hline \multirow[t]{2}{*}{ Fatores } & \multicolumn{2}{|c|}{ Prematuros } & \multirow[t]{2}{*}{ Valor $p$} \\
\hline & Sem ROP & Com ROP & \\
\hline Doenças pulmonares & 79 (34,6\%) & $31(53,4 \%)$ & 0,0041 \\
\hline Oxigenioterapia & $100(43,9 \%)$ & $44(75,9 \%)$ & $<0,0000$ \\
\hline Transf. sangüínea & $21(9,2 \%)$ & $16(27,6 \%)$ & 0,0002 \\
\hline Alter. SNC & $2(0,9 \%)$ & $3(5,2 \%)$ & 0,0259 \\
\hline Infecções graves & $12(20,7 \%)$ & $23(10,1 \%)$ & 0,0278 \\
\hline Apgar $<7$ no $1^{\circ}$ min & $67(35,3 \%)$ & $20(52,6 \%)$ & 0,0442 \\
\hline Fonte: Pesquisa de camp & & & \\
\hline
\end{tabular}

Quadro 2. Fatores entre os prematuros sem significado estatístico para a ROP. Maternidade Darcy Vargas - Joinville (SC). 1992 - 1999

\begin{tabular}{lrrr} 
Fatores & \multicolumn{2}{c}{ Prematuros } & Valor $\mathbf{p}$ \\
\cline { 2 - 4 } & \multicolumn{1}{c}{ Sem ROP } & \multicolumn{1}{c}{ Com ROP } & \\
Apgar no 5o minuto & $<=20(10,4 \%)$ & $<7=7(18,4 \%)$ & 0,1613 \\
Fototerapia & $64(28,1 \%)$ & $15(25,9 \%)$ & 0,7370 \\
Anomalias congênitas & $22(9,6 \%)$ & $4(6,9 \%)$ & 0,5150 \\
Doenças cardíacas & $7(3,1 \%)$ & $3(5,2 \%)$ & 0,4365 \\
Alterações do & $3(1,3 \%)$ & $1(1,7 \%)$ & 0,6968 \\
sistema digestivo & & & \\
D. metabólicas & $11(4,8 \%)$ & $6(10,3 \%)$ & 0,1124 \\
Toxoplasmose & $1(0,4 \%)$ & $1(1,7 \%)$ & 0,2942 \\
Outras doenças & $56(24,6 \%)$ & $11(19,0 \%)$ & 0,7258 \\
Fonte: Pesquisa de campo & & & \\
\hline
\end{tabular}

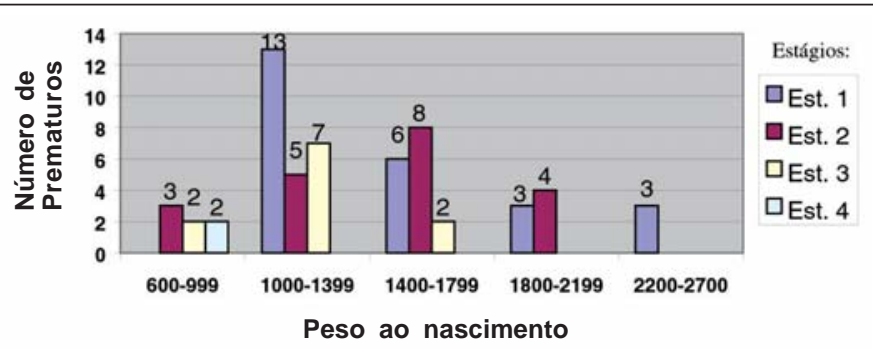

Gráfico 2 - Retinopatia da prematuridade nos diferentes estágios versus peso ao nascimento na Maternidade Darcy Vargas - Joinville (SC). 1992 - 1999

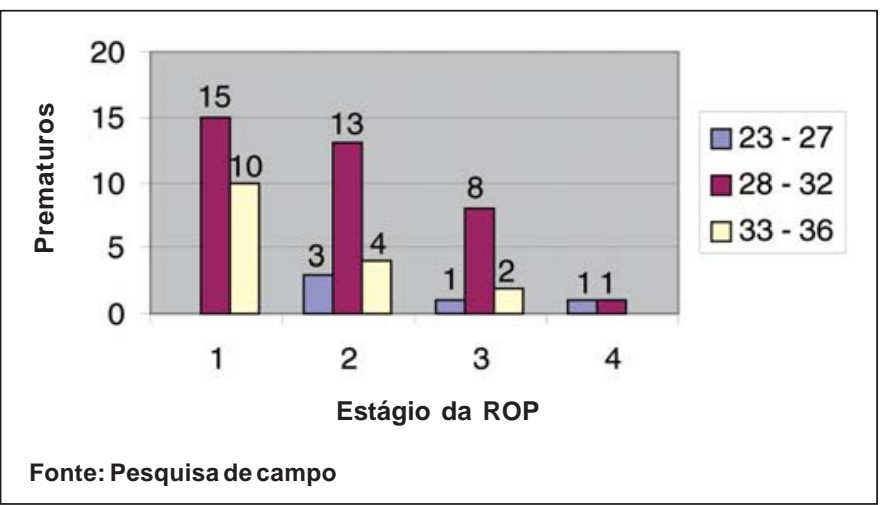

Gráfico 3 - Retinopatia da prematuridade nos diferentes estágios versus idade gestacional, Maternidade Darcy Vargas, Joinville (SC). 1992 - 1999 
laserterapia, a prematura 470 de PN 900 g e IG de 30 semanas e o prematuro 598 com PN 715 g e IG 26 semanas.

\section{DISCUSS ÃO}

Até 1992 Joinville não contava com nenhum acompanhamento oftalmológico de rotina em nenhuma maternidade. Considerando-se a importância da ROP e conhecendo-se os riscos que os prematuros estavam correndo, foi desenvolvido o presente estudo para avaliar a frequiência da retinopatia da prematuridade na Maternidade Darcy Vargas, maior maternidade da cidade com uma média de 6.500 nascimentos/ano e média de 300 prematuros/ano. Esta maternidade atende parturientes e neonatos da rede pública, conveniada e particular, da cidade e da região norte de Santa Catarina, e é referência em todo o Estado.

Os prematuros representaram $3 \%$ do total de nascimentos, durante o período de junho de 1992 a junho de 1999. Dezoito por cento deles foram a óbito antes da alta hospitalar e em $14 \%$ isto aconteceu antes dos sete dias de vida, apesar de toda a tecnologia envolvida. A frequiência de óbitos, das primeiras 24 horas ao sétimo dia de vida, é alta no grupo de prematuros. A literatura mostra que as crianças que nascem com muito baixo peso ou com peso muito acima do esperado para a idade gestacional, morrem em maiores proporções no $1^{\circ}$ dia de $\operatorname{vida}^{(20-21)}$.

Observou-se uma perda de $39 \%$ entre os prematuros que saíram da maternidade e os que chegaram a ser catalogados no serviço de acompanhamento ambulatorial. Entre as possíveis causas têm-se as inúmeras internações a que estes prematuros são submetidos após a alta da maternidade em outros hospitais, óbitos não comunicados, residência em outro município, dificuldade financeira e outros. Foi tentado sanar este problema, no início deste trabalho, com comunicação em rádio, telegramas e cartas, sem ser alcançada a resposta esperada. A perda de casos se repete nos retornos ambulatoriais, podendo as causas serem as mesmas. O pediatra quando vê o cartão de retorno ou as anotações na caderneta de saúde, reencaminha, mas o período crítico para detecção da ROP pode ter passado. Neste estudo foram excluídos 423 prematuros dos 709 que entraram no banco de dados, por não cumprirem os critérios de inclusão. Além do peso e idade gestacional, foi considerada a necessidade de, no mínimo, três consultas de acompanhamento oftalmológico, porque muitas vezes no primeiro exame não se constata a retinopatia e nos exames subseqüentes ela aparece ${ }^{(16)}$. Todos deveriam ter no mínimo 6 meses de idade até dezembro de 1999, porque neste período (180 dias) a retina já cumpriu seu crescimento tendo ou não desenvolvido ROP.

Existem várias opiniões sobre a dilatação. Alguns autores defendem tropicamida $1 \%$ outros usam ciclopentolato diluído, fenilefrina a $0,25 \%$ com tropicamda $0,5 \%{ }^{(12-14,22-24)}$. Isto é importante em cidades onde as distâncias são grandes e o oftalmologista não tem horário certo de chegada na Unidade.

No início, foi obedecido o protocolo de tropicamida a $0,5 \%$ e fenilefrina a $0,25 \%$. Observou-se com o tempo que a dilatação era difícil tanto com a mistura quanto sem a mesma. Nestes casos optava-se por repetir o exame num outro dia, evitando-se a mistura.

Na maioria das unidades neonatais quem faz a dilatação é a enfermeira. No caso da Maternidade Darcy Vargas é a auxiliar de oftalmologia quem prepara os colírios e os aplica. Sendo sempre a mesma pessoa, há mais uniformidade na rotina de aplicação.

O critério de "screening" também é motivo de discussão. Muitos autores, no início deste estudo, já defendiam que o peso ao nascimento deveria ser abaixo de $1.500 \mathrm{~g}$ e idade gestacional menor que 32 semanas $^{(1,5,9-10,23,25-27)}$. Neste estudo foi determinada idade gestacional menor ou igual a 36 semanas e peso ao nascer menor ou igual a $1.600 \mathrm{~g}$. Os prematuros com idade gestacional de 36 semanas e 3 dias, era arredondada para 36 semanas. A idade gestacional prevalecia sobre o peso. Isto é, se a criança nascesse com $2.000 \mathrm{~g}$ e idade gestacional de 35, era incluída no estudo. Antes desta pesquisa não se sabia se a ROP existia entre os prematuros nascidos na MDV. Além disso, não se conhecia a faixa de peso e idade gestacional onde a retinopatia era mais prevalente. Por este motivo o limite de screening foi ampliado, e o fator mais importante foi a idade gestacional abaixo de 37 semanas.

Esta dificuldade, sobre que limites adotar na seleção do prematuro que necessita ser avaliado, é observada em outros trabalhos mais recentes no Brasil ${ }^{(14,22,28-29)}$.

Alguns autores defendem que o tempo para o primeiro exame pode ser 4 a 6 semanas de vida ${ }^{(22,28,30)}$. Outros defendem que o primeiro exame deve ser feito entre 31 ou 32 semanas da idade pós-concepção(5).

Observando a literatura, este estudo adotou o primeiro exame entre 4 a 6 semanas de vida ${ }^{(17)}$.

Preferiu-se não estudar o grupo racial. Apesar de este item estar no banco de dados, existe uma dificuldade muito grande em separar os prematuros dentro da miscigenação brasileira. Existem, na literatura, trabalhos que parecem demonstrar uma maior tendência da ROP nos brancos ${ }^{(15,21)}$, enquanto outros autores não encontraram significado estatístico em brancos e não brancos ${ }^{(10,31)}$. A terminologia branco e não branco seria talvez mais adequada para determinação deste fator em próximos trabalhos.

Cunha et al., (1984) num estudo retrospectivo de ROP, avaliando fichas de pacientes que procuravam o serviço por leucocoria ou baixa visão, foram separados para estudo 40 pacientes com ROP. Nestes, os autores observaram uma incidência maior no sexo feminino $(63,1 \%)^{(32)}$.

No presente estudo o fator sexo não teve significado estatístico para a doença em questão, assim como aconteceu no estudo de alguns autores ${ }^{(22,28)}$.

A perda de acompanhamento dos prematuros, ou por óbito ou por descontinuidade de seguimento pelas razões já comentadas, impossibilitou o cálculo da prevalência de ROP dos prematuros da MDV. Estes prematuros são provenientes da cidade de Joinville e norte catarinense, esporadicamente são encaminhadas mães de gestação de risco de outras regiões do Estado. 
60 Prevalência de retinopatia da prematuridade em prematuros atendidos no período de 1992-1999 em Joinville (SC):

avaliação de riscos associados - "screening"

A busca de uma incidência, que reflita a realidade de Santa Catarina e do Brasil, necessita de uma maior organização de dados e um maior envolvimento das nossas organizações de saúde pública. Pois como foi afirmado no I Workshop de Retinopatia da Prematuridade, ocorrido em outubro de 2002 no Rio de Janeiro, o Brasil não dispõe de nenhum programa de diagnóstico ou tratamento a nível nacional e conta apenas com iniciativas isoladas em algumas unidades públicas e privadas, que utilizam diferentes critérios de diagnóstico e tratamento ${ }^{(6)}$.

A prevalência da retinopatia foi de $20 \%$, considerando-se os 286 prematuros que obedeceram aos critérios de inclusão do estudo (Gráficos 1, 2, 3). Moraes (1996), observou ROP em 27,8\% das crianças examinadas e Graziano et al. (1994), 29,9\%. À semelhança do que aconteceu na tese de Moraes, observou-se neste estudo a presença de ROP em idade gestacional e pesos ao nascimento acima do encontrado na literatura internacional. Outros autores também chamaram a atenção sobre a presença de ROP nos pesos acima de $2.000 \mathrm{~g}$, quando existe perda sangüínea importante no pré-natal e necessidade de cirurgia sob anestesia geral ${ }^{(14,22,33)}$.

A retinopatia dita limiar, observada no presente trabalho, concentrou-se abaixo de 1.399 g e idade gestacional inferior a 33 semanas (houve apenas um caso com 35 semanas IG). Esta constatação é importante, pois ajuda a desenhar um programa mais objetivo de "screening" evitando exames desnecessários; concentrando, assim, materiais e pessoal especializado nas crianças que realmente carecem de atenção.

Pode haver uma variação local na incidência da retinopatia e talvez seja esta a melhor explicação para os diferentes achados. Outra explicação para estas diferenças pode estar relacionada com a maneira de se efetuar o exame ${ }^{(29)}$. Por último poderá estar relacionada à sobrevida dos pacientes de risco, variação nas crianças consideradas de baixo peso em relação à idade gestacional (PIG). Esta última variável não foi separada neste estudo e fica aqui uma sugestão para futuras investigações.

A pesquisa vem ao encontro de inúmeros trabalhos já publicados quando demonstra altíssimo significado estatístico para peso ao nascimento $(\mathrm{p}<0,0001)$, oxigenioterapia $(\mathrm{p}<0,0000)$, idade gestacional $(\mathrm{p}=0,0006)$ e transfusão de sangue $(\mathrm{p}=0,0002)^{(14,29,34)}$.

O grupo I apresentou 228 prematuros e o grupo II ficou formado por 58 prematuros.

A prevalência da ROP no referido estudo no período de 1992 a 1999 foi de $20 \%$.

Os fatores relacionados à criança pré-termo que predispõem à retinopatia da prematuridade são idade gestacional, peso ao nascimento, doenças pulmonares, oxigenioterapia, alterações do sistema nervoso central, transfusão sangüínea, infeções graves e Apgar menor que 7 no primeiro minuto.

\section{CONCLUSÃO}

A retinopatia dita limiar, observada no presente trabalho, concentrou-se abaixo de 1.399 g e idade gestacional inferior a 33 semanas (houve apenas um caso com 35 semanas IG).
Conclui-se que o melhor "screening" será feito examinando prematuros abaixo de $1.400 \mathrm{~g}$ e com idade gestacional menor que 33 semanas.

\section{AGRADECIMENTOS}

Agradeço aos professores da Universidade Federal do Paraná, aos médicos e enfermeiros da Maternidade Darcy Vargas e aos colegas de trabalho do Hospital de Olhos Sadalla Amin Ghanem, por esta inestimável colaboração.

\section{ABSTRACT}

Purposes: To evaluate the prevalence of retinopathy of prematurity in premature babies examined at the "Hospital de Olhos Sadalla Amin Ghanem", coming from the "Maternidade Darcy Vargas" during the period from June 1992 to June 1999. To describe the risk factors that cause a predisposition to develop retinopathy of prematurity in the "Maternidade Darcy Vargas" and criteria to improve screening. Methods: 286 premature babies were selected in accordance with the predetermined criteria (gestation period less than 37 weeks, first examination at 4-12 week of age, minimum of 3 ophthalmic examinations before being 180 days old and registration at the "Hospital de Olhos Sadalla Amin"). An analysis of the total group of premature babies was carried out, being classified into Group 1 (without retinopathy of prematurity) and Group 2 (with retinopathy of prematurity) and the factors related to presence or not of retinopathy of prematurity in the premature babies were described. In order to evaluate the frequency of retinopathy of prematurity and factors associated with its occurrence, the Chi-Squared or the Fisher Exact tests were used. Margin of error 5\%. Results: 228 premature babies did not present sings of retinopathy of prematurity (group 1) and 58 presented signs (group 2). The prevalence of retinopathy of prematurity was $20 \%, 9 \%$ being in stage $1 ; 7 \%$ in stage 2 ; $4 \%$ in stage 3 and $1 \%$ in stage $4 \mathrm{a}$. The most involved factors were: birth weight $(\mathrm{p}<0.0001)$, oxygen therapy $(\mathrm{p}<0.0000)$, birth age $(p=0.0006)$, lung disease $(p=0.0041)$, blood transfusion $(\mathrm{p}=0.0002)$, central nervous system alterations $(\mathrm{p}=0.0259)$, serious infections $(\mathrm{p}=0.0278)$ and Apgar less than 7 in the first minute $(\mathrm{p}=0.0442)$. The premature babies that most needed treatment weighed less than 1,399 $\mathrm{g}$ and were 33 weeks old. Conclusion: According to the data the prevalence of retinopathy of prematurity was $20 \%$. The premature babies' risks of retinopathy of prematurity were concentrated on weight below 1,400 $\mathrm{g}$ and age of 33 weeks. Bigger premature babies should be observed when one or more of the following factors are present: oxygen therapy, blood transfusion, lung disease, central nervous system alterations, serious infections and Apgar less than 7 in the first minute.

Keywords: Retinopathy of prematurity/epidemiology; Infant, premature; Risk factors 


\section{REFERÊNCIAS}

1. Sá LCF. Aspectos atuais da retinopatia da prematuridade. J Pediatr. (Rio de J.). 1990;66(8/9):220-4.

2. Terry TL. Extreme prematurity and fibroblastic over-growth of persistent vascular sheath behind each crystalline lens. Am J Ophthalmol. 1942;25:203-4.

3. Owens WL, Owens EW. Retrolental fibroplasia in premature infants. Am J Ophthalmol. 1949;32:1-21.

4. Reese AB, Payne F. Persistence and hyperplasia of the primary vitreous, retrolental fibroplasia. Arch Ophthalmol. 1949;41:527-52.

5. Weakley DR Jr, Spencer R. Current concepts in retinopathy of prematurity, Early Hum Dev. 1992;30(2):121-38.

6. Sociedade Brasileira de Pediatria. Conselho Brasileiro de Oftalmologia. Prevenção de Cegueira Infantil causada por Retinopatia da Prematuridade Estratégia de Exame e Critérios de Triagem. [texto na Internet]. São Paulo: CBO. [citado 2005 Abr 14]. Disponivel em: http://www.cbo.com.br/cbo/ sociedades/pediatria/artigos/DiretrizesexameROPset2004final.pdf

7. Ben-Sira I, Nissenkorn I, Kremer I. Retinopathy of prematurity. Surv Ophthalmol. 1988;33(1):1-16.

8. An international classification of retinopathy of prematurity. The Committee For The Classification off Retinopathy of Prematurity. Arch Ophthalmol. 1984;102(8):1130-4

9. An International classification of retinopathy of prematurity. II. The Classification of retinal detachment. The International Committee for the Classification of the late Stage of Retinopathy of Prematurity Arch Ophthalmol. 1987;105 (7):906-12. Erratum in: Arch Ophthalmol. 1987;105(11):1498.

10. Purohit DM, Ellison C, Zierler S, Miettinen OS, Nadas AS. Risk factors for retrolental fibroplasia: experience with 3025 premature infants. National Collaborative Study of Patent Ductus Arteriosus in Premature Infants. Pediatrics $1985 ; 76(3): 339-44$.

11. Moore A. Retinopathy of prematurity. In: Taylor D. Pediatric ophthalmology. Boston: Blackwell Scientific;1990. p.365-76.

12. Bolt B, Benz B, Koerner F, Bossi E. A mydriatic eye-drop combination without systemic effects for premature infants. J Pediatr Ophthalmol Strabismus. 1992;29(3):157-62. Comment in: J Pediatr Ophthalmol Strabismus. $1994 ; 31(2): 130$

13. Wheatcroft S, Sharma A, McAllister J. Reduction in mydriatic drop size in premature infants. Br J Ophthalmol. 1993;7(6):364-5.

14. Graziano RM. Retinopatia da prematuridade - contribuição ao estudo da ocorrência e análise dos fatores de risco [tese]. São Paulo: Faculdade de Medicina da Universidade de São Paulo; 1994.

15. Palmer EA, Flynn JT, Hardy RJ, Phelps DL, Phillips CL, Schaffer DB, et al. Incidence and early course of retinopathy of prematurity. The Cryotherapy for Retinopathy of Prematurity Cooperative Group. Ophthalmology. 1991;98(11): 1628-40.

16. Fledelius HC, Scherfig E. Eye surgery in severe retinopathy of prematurity: Experience from the Copenhagen University Eye Clinic of Rigshospitalet 1986-91, with emphasis on cryotherapy. Acta Ophthalmol Suppl. 1993;(210): 68-71.

17. Multicenter trial of cryotherapy for retinopathy of prematurity. Preliminary results. Cryotherapy for Retinopathy of Prematurity Cooperative Group Arch Ophthalmol. 1988;106(4):471-9.

18. Hindle NW. Results of cryotherapy at different locations for retinopathy of prematurity. J Pediatr Ophthalmol Strabismus. 1993;30(1):19-23.

19. Flage T. Cryotherapy for retinopathy of prematurity, International perspectives and some recommendations for treatment. Acta Ophthalmol Suppl. 1993; (210):66-7.

20. Barbieri MA, Zucoloto S, Gomes UA, Gurgel RQ, Cipolotti R, Kajiwara JK, et al. Estudo do comportamento das taxas de mortalidade fetal, perinatal e neonatal em um hospital universitário. II. Inter-relações de causa básica de morte, obtida em necrópsia com peso ao nascer e idade no óbito. J Pediatr. (Rio de J.). 1988;64(6):205-10.

21. Costeloe K, Hennessy E, Gibson AT, Marlow N, Wilkinson AR. The EPI Cure study: outcomes to discharge from hospital for infants born at the threshold of viability. Pediatrics. 2000;106(4):659-71. Comment in: Arch Dis Child Fetal Neonatal Ed. 2003;88(3):F256.

22. Moraes NSB. Retinopatia da prematuridade: contribuição para o diagnostico precoce e detecção das doenças oculares associadas [tese]. São Paulo: Universidade Federal de São Paulo; 1996.

23. Laws DE, Morton C, Weindling M, Clark D. Systemic effects of screening for retnopathy of prematurity. Br J Ophthalmol. 1996;80(5):425-8.

24. Garcia CAA, Gomes AHB, Barbosa MFA, Rocha MLR, Uchoa RAC. Avaliação oftalmológica e fatores de risco da retinopatia da prematuridade na Maternidade Escola Januário Cicco, Natal - RN. Rev Bras Oftalmol. 2001;60(7):489-94.

25. Brownn DR, Biglan AW, Stretavsky MA. Screening criteria for the detection of retinopathy of prematurity in patients in a neonatal intensive care unit. J Pediatr Ophthalmol Strabismus. 1987;24(5):212-14.

26. Fledelius HC, Rosenberg T. Retinopathy of prematurity. Where to set screening limits? recommendations based on two Danish surveys. Acta Paediatr Scand. 1990;79(10):906-10.

27. Robinson R, O’Keefe M. Follow-up study on premature infants with and without retinopathy of prematurity. Br J Ophthalmol. 1993;77(2):91-4.

28. Graziano RM, Leone CR, Cunha SL, Pinheiro AC. Prevalência da retinopatia da prematuridade em recém-nascidos de muito baixo peso. J Pediatr (Rio de J.). 1997;73(6):377-82

29. Motta MMS. Retinopatia da prematuridade limiar em crianças submetidas à terapia com surfactante endotraqueal [tese] São Paulo: Universidade Federal de São Paulo; 1997.

30. Moraes NSB, Farah ME, Bonomo PP, Almeida MFB. Laser de diodo versus crioterapia no tratamento da retinopatia da prematuridade: estudo comparativo. Arq Bras Oftalmol. 1997;60(6):635-8.

31. Hussain N, Clive J, Bhandari V. Current incidence of retinopathy of prematurity, 1989-1997. Pediatrics. 1999;104(3):e26.

32. Cunha RP, Cunha MC, Abreu M, Ferreira AA. Reinopatia da prematuridade (fibroplasia retrocristaliniana). Relato de 40 casos (1980 - 1983). Arq Bras Oftalmol. 1984;47(3):111-14

33. Jandeck C, Kellner U, Kossel H, Bartsch M, Versmold HT, Foerster MH. Retinopathy of prematurity in infants of birth weight $>2.000 \mathrm{~g}$ after haemorragic schock at birth. Br J Ophthalmol, 1996;80(8):728-31.

34. Flynn JT, Bancalari E, Bachynski BN, Buckley EB, Bawol R, Goldberg R, et al. Retinopathy of prematurity: diagnosis, severity, and natural history. Ophthalmology. 1987;94(6):620-9.

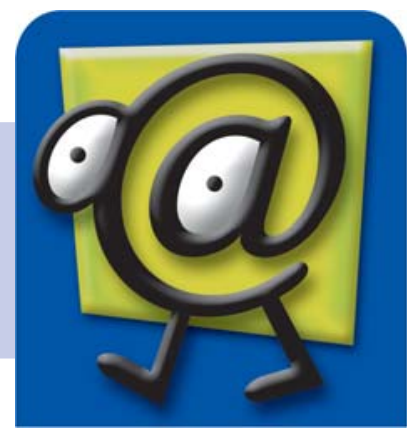

